\title{
Risk Factors of Poor Pregnancy Outcome in Living Kidney Donors in UK
}

\author{
Shanmugapriya Basker \& Sunil Daga \\ NHS Digital, University of Manchester, UK \\ Correspondence to s.basker@nhs.net
}

cite as: Basker S \& Daga S. Risk factors of poor pregnancy outcome in living kidney donors in UK. The Physician 6(1)c13 DOI: 10.38192/1.6.1.c13

\section{Background:}

Despite thousands of kidney donations every year across the world, there is decline in donations particularly within the BAME (Black Asian Minority and Ethnic community). Young female faces a dilemma of future pregnancy when considering decision to donate a kidney. The study aims to describe the risk in pregnancy based on ethnicity, gender and regional variations.

Method:

This project uses Hospital Episode Statistics (HES) Admitted Patient Care (APC) data from 1997/8 to 2017/18 (20 years) with pseudo-anonymous data filtered by kidney donation codes.The dataset was extremely imbalanced with total of 420 records of which 279 negative class (no risk) and 141 (risk) positive class From available 128 columns Mothers Age, Ethnicity (Black, White, South Asian and Other), Deprivation Index ( Low, Medium and High Deprivation Index), Provider Centre (Low, Medium and High provider), hypertension before or at donation, Smoking, Obesity, Risk After Donation Days were taken as features to analyse the data.

Results:

Mother's Age at delivery, smoking and obesity have been outlined with p-value less than 0.05 and proved to be most significant feature. The Odds Ratio showed, 1 unit increase is 1.067 times more likely to cause risk, whereas 10 unit increase is 9 times more likely to have risk in pregnancy after donation. With smoking, it was 2 times more likely to cause risk in pregnancy. The analysis showed Obesity would cause 8 times more risk in pregnancy after donation.

Conclusions:

Mother's age at pregnancy, smoking and obesity were the significant features that would cause risk in pregnancy. Ethnicity has no relevance or influence at all. 